\title{
Estimating the Contribution of Acute Gastroenteritis to the Overall Prevalence of Irritable Bowel Syndrome
}

\author{
Eric D Shah, ${ }^{1}$ Mark S Riddle, ${ }^{2}$ Christopher Chang $^{3}$ and Mark Pimentel ${ }^{3 *}$ \\ ${ }^{1}$ School of Medicine, Texas Tech University Health Sciences Center, Lubbock, Texas, USA; ${ }^{2}$ Enteric Diseases Department, Naval Medical \\ Research Center, Silver Spring, Maryland, USA; and ${ }^{3}$ GI Motility Program, Cedars-Sinai Medical Center, Los Angeles, California, USA
}

\begin{abstract}
Background/Aims
Recent studies reveal that acute gastroenteritis can precipitate irritable bowel syndrome (IBS) symptoms leading to the concept of post-infectious IBS. However, the overall contribution of gastroenteritis to the total IBS prevalence is unknown. In this exercise we try to estimate the contribution of gastroenteritis in IBS using the published literature and a longitudinal approach.
\end{abstract}

\section{Methods}

Existing literature was reviewed to determine the incidence of IBS after gastroenteritis, the rate of remission over time, data on rates of gastroenteritis in a given population and any patterns of resistance to these effects in human populations. This produced 3 models. The first assumed all humans were susceptible to gastroenteritis and its ability to produce IBS. The second assumed (using meta-analysis data) that $90 \%$ of humans in a given outbreak would be resistant to this effect. The third model used a high gastroenteritis exposure rate as might be seen in military deployment.

\section{Results}

In model 1, the prevalence was unrealistically high with an eventual steady state of $43.6 \%$ of the population affected by IBS. In a very conservative approach (model 2), steady state was achieved after 10 years to an overall prevalence of $8.9 \%$. Interestingly, based on a high 1 year exposure rate such as military deployment, the maximum prevalence (steady state) was reached before 1 year suggesting high risk.

\section{Conclusions}

Although hypothetical in approach, based on conservative estimates in existing literature the contribution of gastroenteritis to the overall prevalence of IBS is substantial.

(J Neurogastroenterol Motil 2012;18:200-204)

Key Words

Gastroenteritis; Irritable bowel syndrome; Prevalence

Received: November 10, 2011 Revised: December 13, 2011 Accepted: December 22, 2011

(c) This is an Open Access article distributed under the terms of the Creative Commons Attribution Non-Commercial License (http://creativecommons org/licenses/by-nc/3.0) which permits unrestricted non-commercial use, distribution, and reproduction in any medium, provided the original work is properly cited.

*Correspondence: Mark Pimentel, MD, FRCP(C)

Director, GI Motility Program, Cedars-Sinai Medical Center, 8730 Alden Drive, Suite 225E, Los Angeles, California 90034, USA

Tel: +1-310-423-6143, Fax: +1-310-423-8356, E-mail: pimentelm@cshs.org

Financial support: Cedars-Sinai Medical Center has a licensing agreement with Salix Pharmaceuticals.

Conflicts of interest: Mark Pimentel serves as a consultant for Salix Pharmaceuticals. 


\section{Introduction}

Irritable bowel syndrome (IBS) is a common and debilitating disease process, yet the precise pathophysiology of IBS remains unknown. In recent years, data are continuing to accumulate which suggest that gut bacteria play a role in IBS symptom development. In one bacterial hypothesis, it is suggested that there is an altered distribution of bacteria in the gut. ${ }^{1,2} \mathrm{~A}$ second bacterial hypothesis suggests that IBS arises from an episode of acute gastroenteritis involving a viral, bacterial or other infection in a susceptible host. Two meta-analyses of the studies to date found that approximately $10 \%$ of subjects who experience acute gastroenteritis subsequently develop IBS. ${ }^{3,4}$ Of note, many of the specific pathogens which have been shown to increase the risk of post-infectious IBS (PI-IBS) are also common among domestically acquired infections (eg, Campylobacter and Salmonella).

While these meta-analyses provide convincing evidence that acute gastroenteritis can precipitate IBS, the overall contribution of acute gastroenteritis to the total IBS population in the United States (US) is unknown. Large prospective observational studies could be conducted to precisely define the contribution of acute gastroenteritis to the burden of IBS in the community. However, these types of studies would be difficult to design and expensive to attempt at this time. Despite these challenges, determining the contribution of acute gastroenteritis to development of IBS in the population as a whole would be vital to understanding implications in long-term health care cost, impact on food and water safety policies, and even impact on missionary or military deployment policy.

Therefore, to raise awareness to this novel hypothesis, we describe a basic and empiric population-based approach to illustrate the theoretical extent to which acute gastroenteritis could contribute to the development of IBS over time, based on the existing literature surrounding PI-IBS.

\section{Materials and Methods}

To estimate the population-attributable fraction of IBS due to a post-infectious etiology, we employed an empiric steady-state model based on factors of population dynamics, infectious disease incidence, PI-IBS risk, and characteristics of IBS illness and duration derived from currently available published literature. Where uncertainty existed, the most conservative estimate was utilized. The following assumptions and parameter estimates were utilized to support the development of 3 models:
(1) The US population remained $307,000,000 .^{5}$

(2) The incidence rate of infectious gastroenteritis was 76 million $(24.76 \%)$ annually ${ }^{6}$ among the US population and was not affected by age.

(3) The incidence rate of IBS was $10 \%$ among those who contracted acute gastroenteritis. ${ }^{3,4}$

(4) Fifty-eight percent of IBS patients went into complete remission after 6 years ${ }^{7}$ but were then eligible to re-contract new IBS through a new acute gastroenteritis event.

(5) The remaining $42 \%$ of IBS patients remained symptomatic throughout their life and were replaced by unaffected individuals at death.

(6) A lifetime of IBS was calculated as US life expectancy (78 years) less the average age of IBS onset (29 years), since the age distribution of IBS was assumed to be similar to that of the overall US age distribution.

(7) Episodes of acute gastroenteritis occurred at some point during the year.

(8) IBS was contracted at year's end and could resolve at the beginning of any given year.

\section{Model 1: All Individuals in the Population Were Equally at Risk for Development of Irritable Bowel Syndrome}

We applied the model to a population that was initially unaffected by acute gastroenteritis or IBS, and the model was run iteratively for a number of years. The assumption in this model was that all humans had the potential to develop IBS and that there was no genetic, host or environmental resistance to this outcome. The model is as follows:

$$
\begin{gathered}
\left(t-p_{\text {yearn } n-1} \times T\right) \times r_{A G E} \times r_{I B S}+p_{\text {yearn }-1} \times \\
T-\sum_{k=1}^{6}\left[\left(t-p_{\text {year } n-k} \times T\right) \times r_{A G E} \times r_{I B S} \times s \times \frac{1}{6}\right]- \\
p_{\text {year } n}=\frac{T}{T} \times T \times(1-s) \\
n=\text { the year during which each incremental annual IBS }
\end{gathered}
$$
prevalence is calculated, beginning at year zero and increased by one until steady-state is reached (when incremental annual IBS prevalence does not change).

$p=$ the IBS prevalence at the end of the year for which the above equation was calculated (beginning with $p_{\text {year } 0}=0$ ).

$t=$ the total US population at risk to contract IBS $(307,000,000)$

$T=$ the total US population $(307,000,000)$

$r_{A G E}=$ the annual incidence of acute gastroenteritis $(24.76 \%)$ 
$r_{I B S}=$ a person's chance of contracting IBS given a case of gastroenteritis during the year for which the equation is being run $(10 \%)$

$s=$ the published remission rate of IBS at a 6 year follow-up $(58 \%)$

$$
\sum_{k=1}^{6}\left[\left(t-p_{\text {year } n-k} \times T\right) \times r_{A G E} \times r_{I B S} \times s \times \frac{1}{6}\right]
$$

The amortization portion of the expression above functions to account for the published IBS remission rate at a 6 year follow-up. The $58 \%$ remission was amortized equally over the 6 years prior.

$$
p_{\text {year } n-50} \times T \times(1-s)
$$

The above component accounts for patients with lifetime IBS who lose their disease state due to death and are replaced by persons of average age who are re-eligible to contract IBS. To accomplish this, the expression becomes relevant once $\mathrm{n}$ equals 50 (78 year lifespan, less age 29 average onset of IBS, plus one to account for IBS starting at the beginning of age 29).

\section{Model 2: Only a Minority of Subjects in the Population Could Develop Irritable Bowel Syn- drome After Gastroenteritis}

A major drawback of Model 1 was the assumption of equal risk of IBS development among individuals. Recent data suggests that there could be genetic pre-determinants to susceptibility of PI-IBS. ${ }^{8}$ This is supported by the fact that in previous meta-analyses, ${ }^{3,4}$ only $10 \%$ of subjects who experienced confirmed gastroenteritis actually developed IBS. Therefore, in this model, the conservative assumption was made that only $10 \%$ of the infected population was susceptible to developing IBS. In essence, $90 \%$ of the population in this model had innate resistance by some mechanism.

For Model 2, the following modifications are made to the assumptions in model 1. Model 1 was designed without any genetic susceptibility, and therefore $r_{I B S}$ was set at $10 \%$ to represent the equal chance of contracting IBS among all 76 million individuals who suffered gastroenteritis in a given year. We have built Model 2 to represent the other extreme of genetic susceptibility. We modified $t$ such that the total US population eligible to contract IBS is only $30,700,000$ ( $10 \%$ of the total US population). In addition, we set $r_{I B S}$ at $100 \%$ such that genetically susceptible individuals would definitely contract IBS if they experienced gastroenteritis. In both models, $10 \%$ of the overall 307 million would be eligible to develop IBS from an initial episode of gastroenteritis. However, in Model 2, the other $90 \%$ of the population could still contract gastroenteritis but would be unable to contract IBS due to lack of genetic susceptibility. The denominator of the equation (T) remains 307 million to account for a prevalence calculation based on the entire US population. $r_{I B S}$ is increased to $100 \%$ and therefore becomes irrelevant to Model 2, since a person with gastroenteritis will contract IBS if they are genetically predisposed to doing so.

\section{Model 3: Assuming a Population With High Incidence of Infectious Gastroenteritis}

Certain individuals, such as active-duty military personnel and religious missionaries, are more prone to developing acute gastroenteritis than those from the regular population. To portray findings among high-risk groups, we applied our previous model of varied individual predisposition to IBS (Model 2) to an active-duty military population whose characteristics were based on data from US. Military deployment studies. In contrast to our normal population in Model 2, the proportion of active-duty individuals who develop acute gastroenteritis was $29 \%$ per month. ${ }^{9}$ We assumed a wartime deployment pattern with a typical initial deployment period of 12 months to a high-risk zone. We then simulated a return to a normal-risk area for the following 9 years.

For model 3, the first year of the model is calculated on a monthly basis using the monthly incidence rate of $29 \%$ (represented by $r_{A G E}$ in high-risk environment) over 12 months from month $\mathrm{m}=0$ through $\mathrm{m}=11$. As in Model 2, $r_{I B S}$ remains $100 \%$ and was therefore irrelevant to this model as well.

$$
\begin{aligned}
& p_{\text {month } m}=\frac{\left(t-p_{\text {month m-1 }} \times T\right) \times r_{A G E \text { in high-risk environment }}+p_{\text {month m-1 }} \times T}{T} \\
& p_{\text {year } 0}=p_{\text {month11 }}
\end{aligned}
$$

Subsequent years of the simulation, beginning with $p_{\text {year } 1 \text {, }}$ were run using the equation and assumptions from model 2.

\section{Results}

Figures 1, 2 and 3 demonstrate the effects of the different models. Interestingly, Model 1 (Fig. 1) appeared invalid since it predicted the overall steady state prevalence of IBS in the US. population to be $43.6 \%$. Because this prediction was non-realistic, Model 1 implied that a given proportion of the population must 


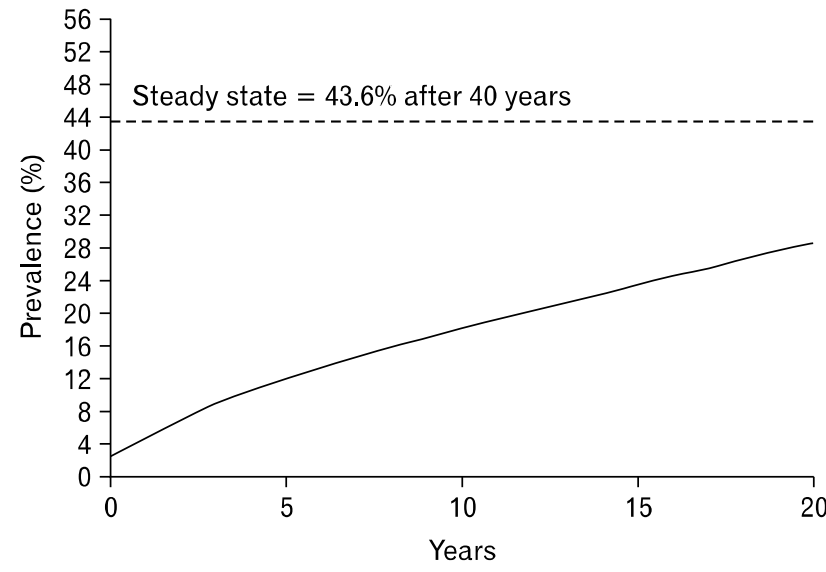

Fiǵure 1. Irritable bowel syndrome prevalence in general population using Model 1 (equal predisposition).

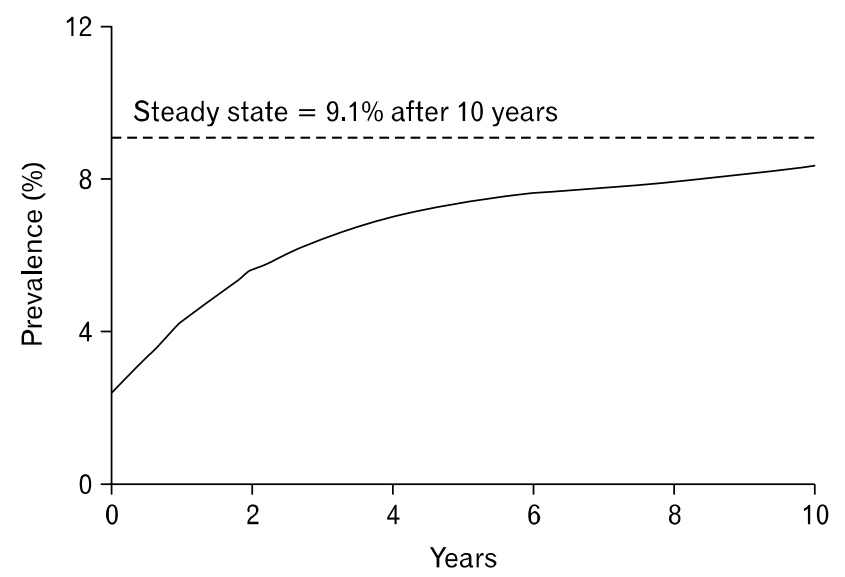

Figure 2. Irritable bowel syndrome (IBS) prevalence in general population using Model 2 (assuming that a majority of subjects are resistant to the development of IBS).

be resistant to the development of IBS.

Model 2 was the most conservative model derived on existing data (Fig. 2). This model was based on an assumption that $90 \%$ of subjects who develop their first episode of gastroenteritis would never develop IBS (at that time and also with any future infection). Yet despite these most conservative assumptions, the model predicted at steady-state that $9 \%$ of the population would have developed IBS from an initial episode of acute gastroenteritis. This was notable, considering the published prevalence of IBS in general.

In Model 3, the only assumption changed was the incidence of acute gastroenteritis to that of troops during deployment (29\% per month). Figure 3 remarkably demonstrated that this population reached an IBS prevalence of almost $10 \%$ after only 12

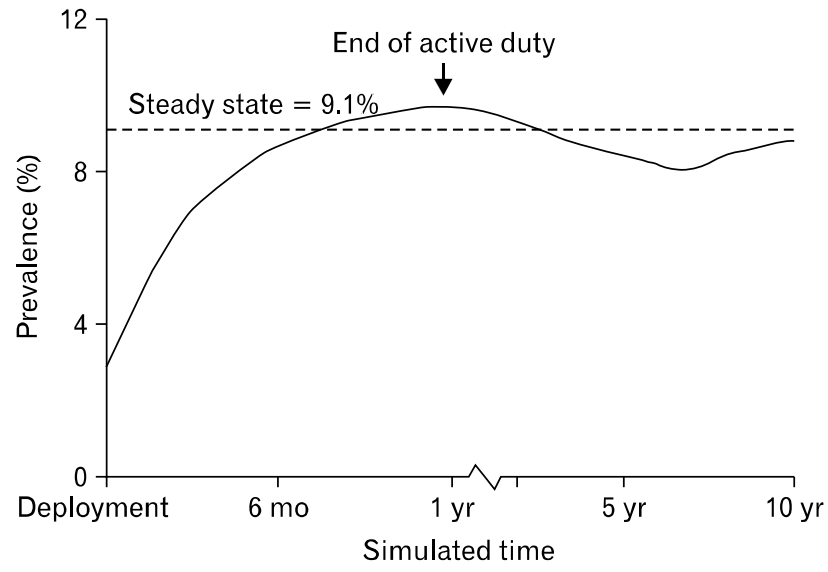

Figure 3. Irritable bowel syndrome development in a high-risk group using Model 3 (active-duty personnel in deployment conditions).

months of deployment. This contrasts to the nearly 10 years to reach this level in Model 2 of a normal population (Fig. 2).

\section{Discussion}

In this hypothetical examination of the contribution of acute gastroenteritis to PI-IBS (and therefore overall IBS), the results suggest a significant long term burden of IBS due to acute gastroenteritis. This was dependent on assumptions derived from published literature.

Irrespective of assumptions used, each of the proposed models has the potential to account for a large number of IBS cases. In fact, the most conservative model (Fig. 2) predicts a steady state prevalence of IBS of around 9\%. This is remarkable, considering that the true prevalence of IBS is published to be $10 \%$ to $15 \% .^{10-15}$ These highly theoretical models suggest that PI-IBS could account for the majority of IBS in the community over time. This becomes even more relevant, as increased incidence of IBS is predicted in populations at higher risk for enteric infection.

Our development of these models is solely intended to suggest a significant impact of acute gastroenteritis in IBS development, not to provide exact projections of current and future IBS prevalence. As such, certain assumptions may have been overly simplified. First, the suggestion that $90 \%$ of humans are resistant to PI-IBS may be overly conservative, and this may be evidenced by increased findings of IBS in military personnel compared to predictions with our simple model of varied individual predisposition to IBS. Second, individuals with repeated enteric infection and also those presenting with fever and bloody stool may experience increased risk of IBS development. ${ }^{3,4,16}$ However, a 
Salmonella outbreak in Spain demonstrated that uniform infection across a population (accounting for varied severity of illness) was associated with a 1-year incidence of new IBS of $12 \% .^{17}$ Therefore, controlling for severity may be unnecessarily complicated.

In contrast to our simple models of population homogeneity, some individuals are subject to certain risk factors and characteristics which affect the probability of developing acute gastroenteritis (and therefore potentially PI-IBS). Individuals who eat mostly home-cooked meals, such as those of certain cultures and age groups, are less likely to contract acute gastroenteritis. Other factors in risk stratification include wealth or religious status, either of which increases the likelihood of travel to areas with high risk of enteric infection. Finally, the contributions of gut microbiota and the mucosal immune system are undoubtedly significant but difficult to characterize at present. Certain individuals may possess a gut flora that can better out-compete a gastrointestinal pathogen inoculum and minimize infection severity, and others may be able to mount a more robust innate or adaptive immune response against infection. Both of these situations would decrease the risk of developing PI-IBS in similar fashion to the varied disease presentations that can occur following exposure to Clostridium difficile. ${ }^{18}$

In conclusion this hypothetical model suggests that PI-IBS may account for the majority of IBS. Further studies are needed to substantiate the short- and long-term impact of acute gastroenteritis on the development of IBS and other functional gastrointestinal motility disorders. While the estimates provided by our models may be high, if founded, they should prompt serious consideration by policy makers and public health authorities on what might be done to mitigate the risk of infectious gastroenteritis through improvements in food and water security and communicable disease control.

\section{Acknowledgements}

We would like to thank the Beatrice and Samuel A Seaver Foundation for their continued support in our research.

Mark Pimentel serves as a consultant for Salix Pharmaceuticals. Cedars-Sinai Medical Center has a licensing agreement with Salix Pharmaceuticals.

\section{References}

1. Posserud I, Stotzer PO, Bjornsson ES, Abrahamsson H, Simren M.
Small intestinal bacterial overgrowth in patients with irritable bowel syndrome. Gut 2007;56:802-808.

2. Lin HC, Pimentel M. Bacterial concepts in irritable bowel syndrome. Rev Gastroenterol Disord 2005;5(suppl 3):S3-S9.

3. Halvorson HA, Schlett CD, Riddle MS. Post-infectious irritable bowel syndrome - a meta-analysis. Am J Gastroenterol 2006;101: 1894-1899.

4. Thabane M, Kottachchi DT, Marshall JK. Systematic review and meta-analysis: The incidence and prognosis of post-infectious irritable bowel syndrome. Aliment Pharmacol Ther 2007;26:535-544.

5. U.S. population projections: National population projections, 2008. Available from: http://www.census.gov/population/www/projections/ summarytables.html (accessed 13 Feb, 2012).

6. Mead PS, Slutsker L, Dietz V, et al. Food-related illness and death in the United States. Emerg Infect Dis 1999;5:607-625.

7. Marshall JK, Thabane M, Garg AX, et al. Eight year prognosis of postinfectious irritable bowel syndrome following waterborne bacterial dysentery. Gut 2010;59:605-611.

8. Villani AC, Lemire M, Thabane M, et al. Genetic risk factors for post-infectious irritable bowel syndrome following a waterborne outbreak of gastroenteritis. Gastroenterol 2010;138:1502-1513.

9. Riddle MS, Tribble DR, Cachafiero SP, Putnam SD, Hooper TI. Development of a travelers' diarrhea vaccine for the military: how much is an ounce of prevention really worth? Vaccine 2008;26: 2490-2502.

10. American College of Gastroenterology Task Force on Irritable Bowel Syndrome, Brandt LJ, Chey WD, et al. An evidence-based systematic review on the management of irritable bowel syndrome. Am J Gastroenterol 2009;104(suppl 1):S1-S35.

11. Talley NJ, Zinsmeister AR, Van Dyke C, Melton LJ 3rd. Epidemiology of colonic symptoms and the irritable bowel syndrome. Gastroenterology 1991;101:927-934.

12. Drossman DA, Li Z, Andruzzi E, et al. US householders survey of functional gastrointestinal disorders: Prevalence, sociodemography, and health impact. Dig Dis Sci 1993;38:1569-1580.

13. Hahn BA, Saunders WB, Maier WC. Differences between individuals with self-reported irritable bowel syndrome (IBS) and IBS-like symptoms. Dig Dis Sci 1997;42:2585-2590.

14. Saito YA, Locke GR, Talley NJ, Zinsmeister AR, Fett SL, Melton LJ 3rd. A comparison of the Rome and Manning criteria for case identification in epidemiological investigations of irritable bowel syndrome. Am J Gastroenterol 2000;95:2816-2824.

15. Thompson WG, Irvine EJ, Pare P, Ferrazzi S, Rance L. Functional gastrointestinal disorders in Canada: first population-based survey using Rome II criteria with suggestions for improving the questionnaire. Dig Dis Sci 2002;47:225-235.

16. Gwee KA, Leong YL, Graham C, et al. The role of psychological and biological factors in postinfective gut dysfunction. Gut 1999;44: 400-406.

17. Mearin F, Perez-Oliveras M, Perello A, et al. Dyspepsia and irritable bowel syndrome after a Salmonella outbreak: one-year follow-up cohort study. Gastroenterol 2005;129:98-104.

18. Kyne L, Warny M, Qamar A, Kelly CP. Asymptomatic carriage of Clostridium difficile and serum levels of IgG antibody against toxin A. N Engl J Med 2000;342:390-397. 